\title{
Fast-collapsing theories
}

\author{
Samuel A. Alexander* \\ Department of Mathematics, the Ohio State University
}

February 2015

\begin{abstract}
Reinhardt's conjecture, a formalization of the statement that a truthful knowing machine can know its own truthfulness and mechanicalness, was proved by Carlson using sophisticated structural results about the ordinals and transfinite induction just beyond the first epsilon number. We prove a weaker version of the conjecture, by elementary methods and transfinite induction up to a smaller ordinal.
\end{abstract}

\section{Introduction}

This is a paper about idealized truthful mechanical knowing agents who know facts in a quantified arithmeticbased language that also includes a connective for their own knowledge $(K(1+1=2)$ is read "I (the agent) know $1+1=2 ")$. It is well known (44, 6], 9], [10, [11, 12]) that such an agent cannot simultaneously know its own truthfulness and its own code. Reinhardt conjectured that, while knowing its own truthfulness, such a machine can know it has some code, without knowing which. This conjecture was proved by Carlson [6]. The proof uses sophisticated structural results from [5] about the ordinals, and involves transfinite induction up to $\epsilon_{0} \cdot \omega$.

We will give a proof of a weaker result, but will do so in an elementary way, inducting only as far as $\omega \cdot \omega$. Along the way, we will develop some machinery that is interesting in its own right. Carlson's proof of Reinhardt's conjecture is based on stratifying knowledge (see [8 for a gentle summary). This can be viewed as adding operators $K^{\alpha}$ for knowledge after time $\alpha$ where $\alpha$ takes ordinal values. Under certain assumptions, theories in such stratified language collapse at positive integer multiples of $\epsilon_{0}$, in the sense that if $\phi$ only contains superscripts $<\epsilon_{0} \cdot n$ ( $n$ a positive integer) then $K^{\epsilon_{0} \cdot n} \phi$ holds if and only if $K^{\epsilon_{0} \cdot(n+1)} \phi$ does. In this paper, collapse occurs at positive integer multiples of $\omega$, hence the name: Fast-collapsing theories.

Our result is weakened in the sense that the background theory of knowledge is weakened. The schema $K(\operatorname{ucl}(K(\phi \rightarrow \psi) \rightarrow K \phi \rightarrow K \psi))$ (ucl denotes universal closure) is weakened by adding the requirement that $K$ not be nested deeper in $\phi$ than in $\psi$ (the unrestricted schema ucl $(K(\phi \rightarrow \psi) \rightarrow K \phi \rightarrow K \psi)$ is preserved, but the knower is not required to know it); the schema $\operatorname{ucl}(K \phi \rightarrow K K \phi)$ is forfeited entirely; and a technical axiom called Assigned Validity (made up of valid formulas with numerals plugged in to their free variables) is added to the background theory of knowledge.

On the bright side, our result is stated in a more general way (we mention in passing how the full unweakened result could also be so generalized, but leave those details for later work). Casually, our main theorem has the following form:

A truthful knowing agent whose knowledge is sufficiently "generic" can be taught its own truthfulness and still remain truthful.

Here "generic" is a specific technical term, but it is inclusive enough to include knowledge that one has some code, thus the statement addresses Reinhardt's conjecture.

In Section 2 we present some preliminaries.

In Section 3 we develop stratifiers, maps from unstratified language to stratified language. These are the key to fast collapse. They debuted in [1] and [3].

*Email: alexander@math.ohio-state.edu 
In Section 4 we discuss uniform stratified theories. A key advantage of stratifiers is that they turn unstratified theories into uniform stratified theories.

In Section 5 we define some notions of genericity of an axiom schema, and establish the genericity of some building blocks of background theories of knowledge.

In Section [6 we state our main theorem and make closing remarks.

\section{Preliminaries}

Definition 1. (Standard Definitions) Let $\mathscr{L}_{\mathrm{PA}}$ be the language $(0, S,+, \cdot)$ of Peano arithmetic and let $\mathscr{L}$ be an arbitrary language.

1. For any $e \in \mathbb{N}, W_{e}$ is the range of the eth partial computable function. The binary predicate $\bullet \in W \bullet$ is $\mathscr{L}_{\mathrm{PA}}$-definable so we will freely act as if $\mathscr{L}_{\mathrm{PA}}$ actually contains this predicate symbol.

2. If an $\mathscr{L}$-structure $\mathscr{M}$ is clear from context, an assignment is a function taking variables into the universe of $\mathscr{M}$.

3. If $s$ is an assignment, $x$ is a variable, and $a \in \mathscr{M}, s(x \mid a)$ is the assignment that agrees with $s$ except that $s(x \mid a)(x)=a$.

4. We define $\mathscr{L}_{\mathrm{PA}}$-terms $\bar{n}(n \in \mathbb{N})$, called numerals, so that $\overline{0}=0$ and $\overline{n+1}=S(\bar{n})$.

5. If $\phi$ is an $\mathscr{L}$-formula, $\mathrm{FV}(\phi)$ is the set of free variables of $\phi$. If $\mathrm{FV}(\phi)=\emptyset$ then $\phi$ is a sentence.

6. If $\phi$ is an $\mathscr{L}$-formula, $x$ is variable, and $u$ is an $\mathscr{L}$-term, $\phi(x \mid u)$ is the result of substituting $u$ for all free occurrences of $x$ in $\phi$.

7. A universal closure of an $\mathscr{L}$-formula $\phi$ is a sentence $\forall x_{1} \cdots \forall x_{n} \phi$. We write ucl $(\phi)$ to denote a universal closure of $\phi$.

8. We use the word theory as synonym for set of sentences.

9. If $T$ is an $\mathscr{L}$-theory and $\mathscr{M}$ is an $\mathscr{L}$-structure, $\mathscr{M} \models T$ means that $\mathscr{M} \models \phi$ for all $\phi \in T$.

10. If $T$ is an $\mathscr{L}$-theory, we say $T \models \phi$ if $\mathscr{M} \models \phi$ whenever $\mathscr{M} \models T$.

11. A valid $\mathscr{L}$-formula is one that holds in every $\mathscr{L}$-structure.

12. For any formulas $\phi_{1}, \phi_{2}, \phi_{3}$, we write $\phi_{1} \rightarrow \phi_{2} \rightarrow \phi_{3}$ to abbreviate $\phi_{1} \rightarrow\left(\phi_{2} \rightarrow \phi_{3}\right)$.

We will repeatedly use the following standard fact without explicit mention: if $\psi$ is a universal closure of $\phi$, then in order to prove $\mathscr{M} \models \psi$, it suffices to let $s$ be an arbitrary assignment and show that $\mathscr{M} \models \phi[s]$.

For quantified semantics we work in Carlson's base logic, defined as follows.

Definition 2. (The Base Logic) A language $\mathscr{L}$ in the base logic is a first-order language $\mathscr{L}_{0}$ together with a set of symbols called operators. Formulas of $\mathscr{L}$ are defined in the usual way, with the clause that whenever $\phi$ is an $\mathscr{L}$-formula and $K$ is an $\mathscr{L}$-operator, $K \phi$ is also an $\mathscr{L}$-formula (and $\mathrm{FV}(K \phi)=\mathrm{FV}(\phi)$ ). Syntactic parts of Definition 1 extend to the base logic in obvious ways. Given such an $\mathscr{L}$, an $\mathscr{L}$-structure $\mathscr{M}$ is a first-order $\mathscr{L}_{0}$-structure $\mathscr{M}_{0}$ together with a function that takes one $\mathscr{L}$-formula $\phi$, one $\mathscr{L}$-operator $K$, and one assignment $s$, and outputs True or False - in which case we write $\mathscr{M} \models K \phi[s]$ or $\mathscr{M} \not K \phi[s]$, respectively - satisfying the following three conditions (where $\phi$ ranges over $\mathscr{L}$-formulas and $K$ ranges over operators):

1. Whether or not $\mathscr{M} \models K \phi[s]$ is independent of $s(x)$ if $x \notin \mathrm{FV}(\phi)$.

2. (Alphabetic Invariance) If $\psi$ is an alphabetic variant of $\phi$, meaning that it is obtained from $\phi$ by renaming bound variables while respecting binding of the quantifiers, then $\mathscr{M} \models K(\phi)[s]$ if and only if $\mathscr{M} \models K(\psi)[s]$. 
3. (Weak Substitution) $\sqrt{1}$ If the variable $y$ is substitutable for the variable $x$ in $\phi$, then $\mathscr{M} \models K \phi(x \mid y)[s]$ if and only if $\mathscr{M} \models K \phi[s(x \mid s(y))]$.

Theorem 3. (Completeness and compactness) Let $\mathscr{L}$ be an r.e. language in the base logic.

1. The set of valid $\mathscr{L}$-formulas is r.e.

2. For any r.e. $\mathscr{L}$-theory $T,\{\phi: T \models \phi\}$ is r.e.

3. There is an effective algorithm, given (a Gödel number for) an r.e. $\mathscr{L}$-theory $T$, to find (a Gödel number for) $\{\phi: T \models \phi\}$.

4. If $T$ is an $\mathscr{L}$-theory and $T \models \phi(\phi$ any $\mathscr{L}$-formula $)$, there are $\tau_{1}, \ldots, \tau_{n} \in T$ such that $\left(\bigwedge_{i} \tau_{i}\right) \rightarrow \phi$ is valid.

Proof. By interpreting the base logic in first-order logic. For details, see [1].

Definition 4. Let $\mathscr{L}_{\mathrm{EA}}$ be the language of Epistemic Arithmetic from [13, so $\mathscr{L}_{\mathrm{EA}}$ extends $\mathscr{L}_{\mathrm{PA}}$ by a unary operator $K$. An $\mathscr{L}_{\mathrm{EA}}$-structure (more generally an $\mathscr{L}$-structure where $\mathscr{L}$ extends $\mathscr{L}_{\mathrm{PA}}$ ) has standard first-order part if its first-order part has universe $\mathbb{N}$ and interprets $0, S,+, \cdot$ in the intended ways.

Definition 5. Suppose $\mathscr{L}$ extends $\mathscr{L}_{\mathrm{PA}}$ and $\phi$ is an $\mathscr{L}$-formula with $\mathrm{FV}(\phi) \subseteq\left\{x_{1}, \ldots, x_{n}\right\}$. For any assignment $s$ into $\mathbb{N}$, we define

$$
\phi^{s} \equiv \phi\left(x_{1} \overline{s\left(x_{1}\right)}\right) \cdots\left(x_{n} \mid \overline{s\left(x_{n}\right)}\right),
$$

the sentence obtained by replacing all free variables in $\phi$ by numerals according to $s$.

Definition 6. For any $\mathscr{L}_{\mathrm{EA}}$-theory $T$, the intended structure for $T$ is the $\mathscr{L}_{\mathrm{EA}}$-structure $\mathscr{N}_{T}$ that has standard first-order part and interprets $K$ so that for any $\mathscr{L}_{\mathrm{EA}}$-formula $\phi$ and assignment $s$,

$$
\mathscr{N}_{T} \models K \phi[s] \text { if and only if } T \models \phi^{s} .
$$

We say $T$ is true if $\mathscr{N}_{T} \models T$.

It is easy to check that the structures $\mathscr{N}_{T}$ of Definition[6]really are $\mathscr{L}_{\mathrm{EA}}$-structures (they satisfy Conditions 1-3 of Definition 21). The following lemma shows that they accurately interpret quantified formulas in the way one would expect.

Lemma 7. For any $\mathscr{L}_{\mathrm{EA}}$-theory $T, \mathscr{L}_{\mathrm{EA}}$-formula $\phi$ and assignment $s$,

$$
\mathscr{N}_{T} \models \phi[s] \text { if and only if } \mathscr{N}_{T} \models \phi^{s} .
$$

Proof. Straightforward induction.

Armed with these definitions, we can make more precise some things we suggested in the introduction. Let $T_{\mathrm{SMT}}$ be the following $\mathscr{L}_{\mathrm{EA}}$-theory ( $\phi$ and $\psi$ range over $\mathscr{L}_{\mathrm{EA}}$-formulas):

1. $\left(E_{1}\right) \mathrm{ucl}(K \phi)$ whenever $\phi$ is valid.

2. $\left(E_{2}\right) \operatorname{ucl}(K(\phi \rightarrow \psi) \rightarrow K \phi \rightarrow K \psi)$.

3. $\left(E_{3}\right) \operatorname{ucl}(K \phi \rightarrow \phi)$.

4. $\left(E_{4}\right) \operatorname{ucl}(K \phi \rightarrow K K \phi)$.

5. The axioms of Epistemic Arithmetic, by which we mean the axioms of Peano Arithmetic with the induction schema extended to $\mathscr{L}_{\mathrm{EA}}$.

6. (Mechanicalness) $\operatorname{ucl}\left(\exists e \forall x\left(K \phi \leftrightarrow x \in W_{e}\right)\right)$ provided $e \notin \mathrm{FV}(\phi)$.

7. $K \phi$ whenever $\phi$ is an instance of lines $1-6$ or (recursively) 7 .

\footnotetext{
${ }^{1}$ Note that the general substitution law, where $y$ is replaced by an arbitrary term, is not valid in modal logic.
} 
Combining lines 6 and 7 yields the Strong Mechanistic Thesis, $K\left(\operatorname{ucl}\left(\exists e \forall x\left(K \phi \leftrightarrow x \in W_{e}\right)\right)\right)$. One of the main results of [6] is that $T_{\mathrm{SMT}}$ is true, that is, $\mathscr{N}_{T_{\mathrm{SMT}}} \models T_{\mathrm{SMT}}$. To establish $\mathscr{N}_{T_{\mathrm{SMT}}} \models E_{3}$, Carlson uses transfinite recursion up to $\epsilon_{0} \cdot \omega$, as well as deep structural properties (from [5]) about the ordinals. That $\mathscr{N}_{T_{\mathrm{SMT}}}$ satisfies lines $2,5,6$, and 7 , is trivial; that it satisfies line 4 follows from the fact that it satisfies lines $1-2$. Line 1 would be trivial if we added the following line to $T_{\mathrm{SMT}}$ :

1b. (Assigned Validity) $\phi^{s}$, whenever $\phi$ is valid and $s$ is any assignment.

Theorems from [6] imply Assigned Validity is already a consequence of $T_{\mathrm{SMT}}$, so this addition is not necessary, however it becomes necessary if (say) line 2 is weakened.

The main result in this paper is that by weakening $E_{2}$, removing $E_{4}$, and adding Assigned Validity, we remove the need to induct up to $\epsilon_{0} \cdot \omega$. Induction up to $\omega \cdot \omega$ suffices, and the computations from [5] can also be avoided. This is surprising because we do not weaken $E_{3}$, the lone schema for which such sophisticated methods were used before.

Definition 8. For any $\mathscr{L}_{\mathrm{EA}}$-formula $\phi$, let $\operatorname{depth}(\phi)$ denote the depth to which $K$ operators are nested in $\phi$, more formally:

- If $\phi$ is an $\mathscr{L}_{\mathrm{PA}}$-formula then $\operatorname{depth}(\phi)=0$.

- If $\phi \equiv K\left(\phi_{0}\right)$ then $\operatorname{depth}(\phi)=\operatorname{depth}\left(\phi_{0}\right)+1$.

- If $\phi \equiv(\rho \rightarrow \sigma)$ then $\operatorname{depth}(\phi)=\max \{\operatorname{depth}(\rho), \operatorname{depth}(\sigma)\}$.

- If $\phi \in\left\{\left(\neg \phi_{0}\right),\left(\forall x \phi_{0}\right)\right\}$ then $\operatorname{depth}(\phi)=\operatorname{depth}\left(\phi_{0}\right)$.

Now let $T_{\mathrm{SMT}}^{w}$ be the $\mathscr{L}_{\mathrm{EA}}$-theory containing the following schemas:

1. $E_{1}$ and $E_{3}$.

2. Assigned Validity: $\phi^{s}$ whenever $\phi$ is valid and $s$ is any assignment.

3. $\left(E_{2}^{\prime}\right) \operatorname{ucl}(K(\phi \rightarrow \psi) \rightarrow K \phi \rightarrow K \psi)$ provided $\operatorname{depth}(\phi) \leq \operatorname{depth}(\psi)$.

4. The axioms of Epistemic Arithmetic.

5. Mechanicalness.

6. $K \phi$ whenever $\phi$ is an instance of lines $1-5$ or (recursively) 6 .

Our main result (obtained by inducting only up to $\omega \cdot \omega$ ) will imply $T_{\text {SMT }}^{w}$ is true.

\section{Stratifiers}

Definition 9. Let $\mathscr{L}_{\omega \cdot \omega}$ be the language obtained from $\mathscr{L}_{\mathrm{PA}}$ by adding operators $K^{\alpha}$ for all $\alpha \in \omega \cdot \omega$. For any $\mathscr{L}_{\omega \cdot \omega}$-formula $\phi$, let

$$
\operatorname{On}(\phi)=\left\{\alpha \in \omega \cdot \omega: K^{\alpha} \text { occurs in } \phi\right\} .
$$

An example of an $\mathscr{L}_{\omega \cdot \omega}$-formula: $\forall x\left(K^{\omega} K^{\omega \cdot 7+2} K^{53} K^{0}(x=0) \rightarrow K^{\omega \cdot 7+3}(x=0)\right)$.

Definition 10. (Stratifiers) For any infinite subset $X \subseteq \omega \cdot \omega$, the stratifier given by $X$ is the function $\bullet+$ that takes $\mathscr{L}_{\text {EA }}$-formulas to $\mathscr{L}_{\omega \cdot \omega}$-formulas in the following way.

1. If $\phi$ is atomic, $\phi^{+} \equiv \phi$.

2. If $\phi$ is $\phi_{1} \rightarrow \phi_{2}, \neg \phi_{1}$, or $\forall x \phi_{1}$, then $\phi^{+}$is $\phi_{1}^{+} \rightarrow \phi_{2}^{+}, \neg \phi_{1}^{+}$, or $\forall x \phi_{1}^{+}$, respectively.

3. If $\phi$ is $K \phi_{0}$, then $\phi^{+} \equiv K^{\alpha} \phi_{0}^{+}$where $\alpha$ is the smallest ordinal in $X \backslash \operatorname{On}\left(\phi_{0}^{+}\right)$.

By a stratifier, we mean a stratifier given by some $X$. By the veristratifier, we mean the stratifier given by $X=\{\omega \cdot 1, \omega \cdot 2, \ldots\}$. If $\bullet+$ is a stratifier and $T$ is an $\mathscr{L}_{\mathrm{EA}}$-theory, $T^{+}$denotes $\left\{\phi^{+}: \phi \in T\right\}$. 
For example, if $\bullet+$ is the veristratifier, then

$$
(K(1=0) \rightarrow K K(1=0))^{+} \equiv K^{\omega}(1=0) \rightarrow K^{\omega \cdot 2} K^{\omega}(1=0) .
$$

Lemma 11. Suppose $\phi$ is an $\mathscr{L}_{\mathrm{EA}}$-formula, $s$ is an assignment into $\mathbb{N}$, and $\bullet+$ is a stratifier. If $\alpha, \beta \in \omega \cdot \omega$ are such that $(K \phi)^{+} \equiv K^{\alpha} \phi^{+}$and $\left(K \phi^{s}\right)^{+} \equiv K^{\beta}\left(\phi^{s}\right)^{+}$, then $\alpha=\beta$.

Proof. By induction.

Lemma 12. Suppose $\phi$ and $\psi$ are $\mathscr{L}_{\mathrm{EA}}$-formulas and $\bullet^{+}$is a stratifier. Let $\alpha, \beta \in \omega \cdot \omega$ be such that $(K \phi)^{+} \equiv K^{\alpha} \phi^{+}$and $(K \psi)^{+} \equiv K^{\beta} \psi^{+}$. Then $\operatorname{depth}(\phi)<\operatorname{depth}(\psi)$ if and only if $\alpha<\beta$.

Proof. By induction.

Definition 13. For any $\mathscr{L}_{\omega \cdot \omega}$-structure $\mathscr{M}$ and stratifier $\bullet^{+}$, let $\mathscr{M}^{+}$be the $\mathscr{L}_{\mathrm{EA}}$-structure that has the same universe and interpretation of $\mathscr{L}_{\mathrm{PA}}$ as $\mathscr{M}$, and that interprets $K$ so that for any $\mathscr{L}_{\mathrm{EA}}$-formula $\phi$ and assignment $s$,

$$
\mathscr{M}^{+} \models K \phi[s] \text { if and only if } \mathscr{M} \models(K \phi)^{+}[s] .
$$

It is easy to check that if $\mathscr{M}$ is an $\mathscr{L}_{\omega \cdot \omega}$-structure then $\mathscr{M}^{+}$really is an $\mathscr{L}_{\mathrm{EA}}$-structure (it satisfies Conditions 1-3 of Definition 22). From now on we will suppress this remark when defining new structures.

Lemma 14. Let $\mathscr{M}$ be an $\mathscr{L}_{\omega \cdot \omega}$-structure, $\bullet+$ a stratifier. For any $\mathscr{L}_{\mathrm{EA}}$-formula $\phi$ and assignment $s$,

$$
\mathscr{M}^{+} \models \phi[s] \text { if and only if } \mathscr{M} \models \phi^{+}[s] .
$$

Proof. A straightforward induction.

Definition 15. For any $\mathscr{L}_{\omega \cdot \omega}$-formula $\phi, \phi^{-}$is the $\mathscr{L}_{\mathrm{EA}}$-formula obtained by changing every operator of the form $K^{\alpha}$ in $\phi$ into $K$. If $T$ is an $\mathscr{L}_{\omega \cdot \omega}$-theory, $T^{-}=\left\{\phi^{-}: \phi \in T\right\}$.

Example 16. $\left(K^{\omega \cdot 8+3} \forall x K^{17}(x=y)\right)^{-} \equiv K \forall x K(x=y)$.

Lemma 17. Let $\bullet+$ be a stratifier. For any $\mathscr{L}_{\mathrm{EA}}$-formula $\phi,\left(\phi^{+}\right)^{-} \equiv \phi$.

Proof. Straightforward.

Definition 18. If $\mathscr{M}$ is an $\mathscr{L}_{\mathrm{EA}}$-structure, let $\mathscr{M}^{-}$be the $\mathscr{L}_{\omega \cdot \omega}$-structure that has the same universe as $\mathscr{M}$, agrees with $\mathscr{M}$ on $\mathscr{L}_{\mathrm{PA}}$, and interprets each $K^{\alpha}$ so that for any $\mathscr{L}_{\omega \cdot \omega}$-formula $\phi$ and assignment $s$,

$$
\mathscr{M}^{-} \models K^{\alpha} \phi[s] \text { if and only if } \mathscr{M} \models K \phi^{-}[s] .
$$

In [6] (Definition 5.4), $\mathscr{M}^{-}$is the stratification of $\mathscr{M}$ over $\omega \cdot \omega$.

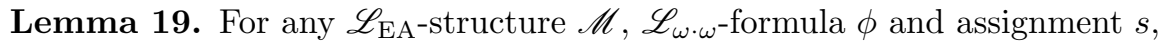

$$
\mathscr{M}^{-} \models \phi[s] \text { if and only if } \mathscr{M} \models \phi^{-}[s] .
$$

Proof. A straightforward induction.

\section{Theorem 20.}

1. For any valid $\mathscr{L}_{\omega \cdot \omega}$-formula $\phi, \phi^{-}$is valid.

2. For any $\mathscr{L}_{\mathrm{EA}}$-formula $\phi$ and stratifier $\bullet^{+}, \phi$ is valid if and only if $\phi^{+}$is valid.

\section{Proof.}

(1) Let $\phi$ be a valid $\mathscr{L}_{\omega \cdot \omega}$-formula. For any $\mathscr{L}_{\text {EA }}$-structure $\mathscr{M}$ and assignment $s$, since $\phi$ is valid, $\mathscr{M}^{-} \models \phi[s]$ and so by Lemma 19, $\mathscr{M} \models \phi^{-}[s]$. By arbitrariness of $\mathscr{M}$ and $s, \phi^{-}$is valid.

$(2, \Rightarrow)$ Assume $\phi$ is a valid $\mathscr{L}_{\text {EA }}$-formula. For any $\mathscr{L}_{\omega \cdot \omega}$-structure $\mathscr{M}$ and assignment $s$, since $\phi$ is valid, $\mathscr{M}^{+} \models \phi[s]$, and $\mathscr{M} \models \phi^{+}[s]$ by Lemma 14, By arbitrariness of $\mathscr{M}$ and $s$, this shows $\phi^{+}$is valid. 


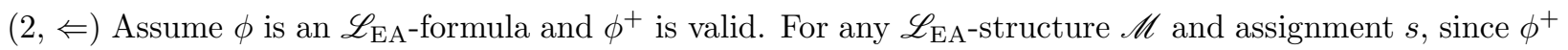
is valid, $\mathscr{M}^{-} \models \phi^{+}[s]$, and $\mathscr{M} \models\left(\phi^{+}\right)^{-}[s]$ by Lemma 19, By Lemma 17, $\mathscr{M} \models \phi[s]$. By arbitrariness of $\mathscr{M}$ and $s, \phi$ is valid.

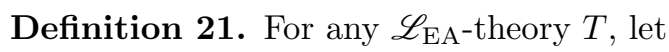

$$
T^{\oplus}=\left\{\phi^{+}: \phi \in T \text { and } \bullet+\text { is a stratifier }\right\} .
$$

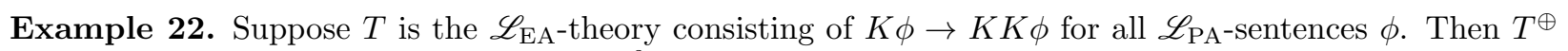

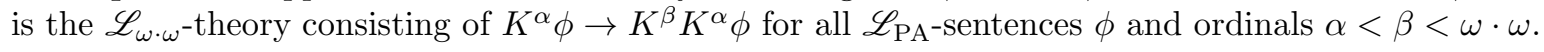

Theorem 23. (Upward proof stratification) For any $\mathscr{L}_{\mathrm{EA}}$-theory $T, \mathscr{L}_{\mathrm{EA}}$-sentence $\phi$, and stratifier $\bullet+$, the following are equivalent.

$$
\text { 1. } T \models \phi . \quad \text { 2. } T^{+} \models \phi^{+} . T^{\oplus} \models \phi^{+} .
$$

This theorem is so-named because it is an upside-down version of a harder theorem that we called [1] proof stratification. In non-upward proof stratification, $T$ and $\phi$ are taken in the stratified language and the theorem states that $T \models \phi$ if and only if $T^{-} \models \phi^{-}$. This uses complicated hypotheses on $T$ and $\phi$. Versions of these hypotheses could be stated in an elementary way, but a priori they might imply $T$ is inconsistent (in which case Theorem 23 is trivial). The only way we know to exhibit consistent theories that satisfy such hypotheses is to exploit the machinery from [5] on the $\Sigma_{1}$-structure of the ordinals.

Proof of Theorem 23. Let $T, \phi$ and $\bullet+$ be as in Theorem 23

$(1 \Rightarrow 2)$ Assume $T \models \phi$. By Theorem 3 there are $\tau_{1}, \ldots, \tau_{n} \in T$ such that $\left(\bigwedge_{i} \tau_{i}\right) \rightarrow \phi$ is valid. By Theorem 20. $\left(\bigwedge_{i} \tau_{i}^{+}\right) \rightarrow \phi^{+}$is valid, showing $T^{+} \models \phi^{+}$.

$(2 \Rightarrow 3)$ Trivial: $T^{+} \subseteq T^{\oplus}$.

$(3 \Rightarrow 1)$ Assume $T^{\oplus} \models \phi^{+}$. By Theorem 3 there are $\tau_{1}, \ldots, \tau_{n} \in T^{\oplus}$ such that $\left(\bigwedge_{i} \tau_{i}\right) \rightarrow \phi^{+}$is valid. By definition of $T^{\oplus}$ there are $\sigma_{1}, \ldots, \sigma_{n} \in T$ and stratifiers $\bullet^{1}, \ldots, \bullet^{n}$ such that each $\tau_{i} \equiv \sigma_{i}^{i}$. By Lemma 17

$$
\left(\left(\bigwedge_{i} \sigma_{i}^{i}\right) \rightarrow \phi^{+}\right)^{-} \equiv\left(\bigwedge_{i} \sigma_{i}\right) \rightarrow \phi
$$

so Theorem 20 guarantees $\left(\bigwedge_{i} \sigma_{i}\right) \rightarrow \phi$ is valid, and $T \models \phi$.

\section{Uniform Theories and Collapsing Knowledge}

Definition 24. Suppose $X \subseteq \omega \cdot \omega$ and $h: X \rightarrow \omega \cdot \omega$. For any $\mathscr{L}_{\omega \cdot \omega}$-formula $\phi$, we define $h(\phi)$ to be the $\mathscr{L}_{\omega \cdot \omega}$-formula obtained by replacing $K^{\alpha}$ by $K^{h(\alpha)}$ everywhere $K^{\alpha}$ occurs in $\phi(\alpha \in X)$. (If $\alpha \notin X$, we do not change occurrences of $K^{\alpha}$ in $\phi$.)

Example 25. Suppose $\alpha_{1}<\cdots<\alpha_{4}$ are distinct ordinals in $\omega \cdot \omega$. Let $X=\left\{\alpha_{2}, \alpha_{3}\right\}$, let $h\left(\alpha_{2}\right)=\alpha_{3}$, $h\left(\alpha_{3}\right)=\alpha_{4}$. Then

$$
h\left(K^{\alpha_{3}} K^{\alpha_{2}} K^{\alpha_{1}}(1=1)\right) \equiv K^{\alpha_{4}} K^{\alpha_{3}} K^{\alpha_{1}}(1=1) .
$$

Definition 26. An $\mathscr{L}_{\omega \cdot \omega}$-theory $T$ is uniform if the following statement holds. For all $X \subseteq \omega \cdot \omega$, for all order-preserving $h: X \rightarrow \omega \cdot \omega$, for all $\phi \in T$, if $\operatorname{On}(\phi) \subseteq X$ then $h(\phi) \in T$.

Example 27. If $T$ contains $K^{1} K^{0}(1=0)$ and $T$ is uniform, then $T$ must contain $K^{\beta} K^{\alpha}(1=0)$ for all $\alpha<\beta \in \omega \cdot \omega$.

Lemma 28. Suppose $\bullet^{+}$is a stratifier, $X \subseteq \omega \cdot \omega, h: X \rightarrow \omega \cdot \omega$ is order preserving, and $\phi$ is an $\mathscr{L}_{\mathrm{EA}}$-formula with $\operatorname{On}\left(\phi^{+}\right) \subseteq X$. There is a stratifier $\bullet^{*}$ such that $\phi^{*} \equiv h\left(\phi^{+}\right)$.

Proof. Let $Y_{0}=\left\{h(\alpha): \alpha \in \operatorname{On}\left(\phi^{+}\right)\right\}, Y=Y_{0} \cup\left\{\beta \in \omega \cdot \omega: \beta>Y_{0}\right\}$, and let $\bullet *$ be the stratifier given by $Y$. By induction, for every subformula $\phi_{0}$ of $\phi, \phi_{0}^{*} \equiv h\left(\phi_{0}^{+}\right)$.

Lemma 29. (Uniformity lemma) For any $\mathscr{L}_{\mathrm{EA}}$-theory $T, T^{\oplus}$ is uniform. 
Proof. Let $X \subseteq \omega \cdot \omega$, let $h: X \rightarrow \omega \cdot \omega$ be order preserving, let $\phi \in T^{\oplus}$, and assume $\operatorname{On}(\phi) \subseteq X$. By definition of $T^{\oplus}, \phi \equiv \phi_{0}^{+}$for some $\phi_{0} \in T$ and some stratifier $\bullet^{+}$. By Lemma 28 there is a stratifier $\bullet^{*}$ such that $h\left(\phi_{0}^{+}\right) \equiv \phi_{0}^{*}$. This shows $h(\phi) \in T^{\oplus}$.

Unfortunately, the range of $\oplus$ does not include every uniform $\mathscr{L}_{\omega \cdot \omega}$-theory. For example, suppose $T$ is the $\mathscr{L}_{\omega \cdot \omega}$-theory consisting of

$$
K^{\alpha}\left(\phi^{+} \rightarrow \psi^{+}\right) \rightarrow K^{\alpha} \phi^{+} \rightarrow K^{\alpha} \psi^{+}
$$

for all $\mathscr{L}_{\mathrm{EA}}$-sentences $\phi$ and $\psi$ and stratifiers $\bullet^{+}$with $\operatorname{On}\left(\phi^{+}\right), \operatorname{On}\left(\psi^{+}\right)<\alpha \in \omega \cdot \omega$. The reader may check that despite being uniform, $T$ is not $T_{0}^{\oplus}$ for any $\mathscr{L}_{\mathrm{EA}}$-theory $T_{0}$.

Definition 30. If $\mathscr{M}$ is an $\mathscr{L}_{\omega \cdot \omega}$-structure, $X \subseteq \omega \cdot \omega$, and $h: X \rightarrow \omega \cdot \omega$, we define an $\mathscr{L}_{\omega \cdot \omega}$-structure $h(\mathscr{M})$ that has the same universe as $\mathscr{M}$, agrees with $\mathscr{M}$ on the interpretation of $\mathscr{L}_{\mathrm{PA}}$, and interprets $K^{\alpha}$ so that for any $\mathscr{L}_{\omega \cdot \omega}$-formula $\phi$ and assignment $s$,

$$
h(\mathscr{M}) \models K^{\alpha} \phi[s] \text { if and only if } \mathscr{M} \models h\left(K^{\alpha} \phi\right)[s] .
$$

Lemma 31. Suppose $\mathscr{M}, X$, and $h$ are as in Definition 30. For any $\mathscr{L}_{\omega \cdot \omega}$-formula $\phi$ and assignment $s$,

$$
h(\mathscr{M}) \models \phi[s] \text { if and only if } \mathscr{M} \models h(\phi)[s] .
$$

Proof. By induction.

We will only need part 1 of the next lemma, we state part 2 for completeness.

Lemma 32. Suppose $\mathscr{M}, X$, and $h$ are as in Definition 30 and $\phi$ is an $\mathscr{L}_{\omega \cdot \omega}$-formula.

1. If $\phi$ is valid then $h(\phi)$ is valid.

2. Assume $h$ is injective. If $\operatorname{On}(\phi) \subseteq X$ and $h(\phi)$ is valid, then $\phi$ is valid.

Proof.

(1) Similar to Theorem 20

(2) If $h(\phi)$ is valid then $h^{-1}(h(\phi))$ is valid by part 1 . Since $\operatorname{On}(\phi) \subseteq X, h^{-1}(h(\phi)) \equiv \phi$.

Definition 33. For any $\mathscr{L}_{\omega \cdot \omega}$-theory $T$ and $\alpha \in \omega \cdot \omega$, let $T \cap \alpha=\{\phi \in T: \operatorname{On}(\phi) \subseteq \alpha\}$ be the subset of $T$ where all superscripts are strictly bounded by $\alpha$.

\section{Example 34.}

- For any $\mathscr{L}_{\omega \cdot \omega}$-theory $T, T \cap 0=\left\{\phi \in T: \phi\right.$ is an $\mathscr{L}_{\mathrm{PA}}$-sentence $\}$.

- For any $\mathscr{L}_{\omega \cdot \omega}$-theory $T, T \cap 1=\left\{\phi \in T: \phi\right.$ is an $\mathscr{L}_{\mathrm{PA}} \cup\left\{K^{0}\right\}$-sentence $\}$.

- For any $\mathscr{L}_{\mathrm{EA}}$-theory $T, T^{\oplus} \cap \omega=\left\{\phi^{+}: \phi \in T\right.$ and $\bullet{ }^{+}$is given by some $\left.X \subseteq \omega\right\}$.

Theorem 35. (The collapse theorem) Let $T$ be a uniform $\mathscr{L}_{\omega \cdot \omega}$-theory. For any $0<n \in \mathbb{N}$ and $\mathscr{L}_{\omega \cdot \omega}$-formula $\phi$ with $\operatorname{On}(\phi) \subseteq \omega \cdot n, T \models \phi$ if and only if $T \cap(\omega \cdot n) \models \phi$.

Proof. The $\Leftarrow$ direction is trivial: $T \cap(\omega \cdot n) \subseteq T$. For $\Rightarrow$, assume $T \models \phi$. By Theorem 3 there are $\tau_{1}, \ldots, \tau_{n} \in T$ such that

$$
\Phi \equiv\left(\bigwedge_{i} \tau_{i}\right) \rightarrow \phi
$$

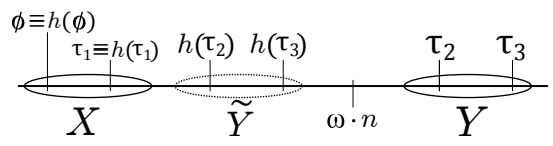

Figure 1: Collapse.

is valid. Let $X=\operatorname{On}(\Phi) \cap(\omega \cdot n), Y=\operatorname{On}(\Phi) \cap[\omega \cdot n, \infty)$, see Fig. 1.

Then $|X|,|Y|<\infty$ and $X \cup Y=\operatorname{On}(\Phi)$. 
Since $|X|<\infty$ and $\omega \cdot n$ has no maximum element, there are infinitely many ordinals above $X$ in $\omega \cdot n$. Thus since $|Y|<\infty$ we can find $\widetilde{Y} \subseteq \omega \cdot n$ such that $X<\tilde{Y}$ and $|\widetilde{Y}|=|Y|$. It follows there is an order preserving function $h: X \cup Y \rightarrow X \cup \tilde{Y}$ such that $h(x)=x$ for all $x \in X$.

By Lemma 32, $h(\Phi)$ is valid. Since $\operatorname{On}(\phi) \subseteq \omega \cdot n$, we have $\operatorname{On}(\phi) \subseteq X$ and $h(\phi) \equiv \phi$. Thus

$$
h(\Phi) \equiv\left(\bigwedge_{i} h\left(\tau_{i}\right)\right) \rightarrow h(\phi) \equiv\left(\bigwedge_{i} h\left(\tau_{i}\right)\right) \rightarrow \phi .
$$

Since $T$ is uniform, each $h\left(\tau_{i}\right) \in T$. In fact, since range $(h) \subseteq \omega \cdot n$, each $h\left(\tau_{i}\right) \in T \cap(\omega \cdot n)$, and the validity of $\left(\bigwedge_{i} h\left(\tau_{i}\right)\right) \rightarrow \phi$ witnesses $T \cap(\omega \cdot n) \models \phi$.

Definition 36. If $T$ is an $\mathscr{L}_{\omega \cdot \omega}$-theory, its intended structure is the $\mathscr{L}_{\omega \cdot \omega}$-structure $\mathscr{M}_{T}$ with standard first-order part that interprets the operators of $\mathscr{L}_{\omega \cdot \omega}$ so that for every $\mathscr{L}_{\omega \cdot \omega}$-formula $\phi$, assignment $s$, and $\alpha \in \omega \cdot \omega$,

$$
\mathscr{M}_{T} \models K^{\alpha} \phi[s] \text { if and only if } T \cap \alpha \models \phi^{s} .
$$

Lemma 37. Suppose $T$ is an $\mathscr{L}_{\omega \cdot \omega}$-theory. For any $\mathscr{L}_{\omega \cdot \omega}$-formula $\phi$ and assignment $s, \mathscr{M}_{T} \models \phi[s]$ if and only if $\mathscr{M}_{T} \models \phi^{s}$.

Proof. By induction.

Recall from Definition 10 that the veristratifier is the stratifier given by $X=\{\omega \cdot 1, \omega \cdot 2, \ldots\}$.

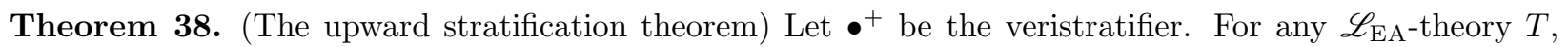
$\mathscr{L}_{\mathrm{EA}}$-formula $\phi$, and assignment $s, \mathscr{N}_{T} \models \phi[s]$ if and only if $\mathscr{M}_{T \oplus} \models \phi^{+}[s]$.

Again, the theorem is so-named because it is an upside-down version of a harder theorem that equates $\mathscr{M}_{T} \models \phi[s]$ with $\mathscr{N}_{T^{-}} \models \phi^{-}[s]$ for stratified $T$ and $\phi$ under more complicated hypotheses.

Proof of Theorem 38. By induction on $\phi$. The only nontrivial case is when $\phi$ is $K \psi$. Then $\phi^{+} \equiv K^{\alpha} \psi^{+}$for some $\alpha$. By definition of the veristratifier, $\alpha=\omega \cdot n$ for some $0<n \in \mathbb{N}$, and $\operatorname{On}\left(\psi^{+}\right) \subseteq \omega \cdot n$. By Lemma 29. $T^{\oplus}$ is uniform, so we can use the collapse theorem (Theorem 35). The following are equivalent.

$$
\begin{aligned}
\mathscr{N}_{T} & \models K \psi[s] \\
T & \models \psi^{s} \\
T^{\oplus} & \models\left(\psi^{s}\right)^{+} \\
T^{\oplus} \cap(\omega \cdot n) & \models\left(\psi^{s}\right)^{+} \\
T^{\oplus} \cap(\omega \cdot n) & \models\left(\psi^{+}\right)^{s} \\
\mathscr{M}_{T^{\oplus}} & \models K^{\omega \cdot n} \psi^{+}[s] .
\end{aligned}
$$

(Upward proof stratification-Theorem 23)

(The collapse theorem-Theorem 351)

(Clearly $\left.\left(\psi^{s}\right)^{+} \equiv\left(\psi^{+}\right)^{s}\right)$

(Definition 36)

Corollary 39. For any $\mathscr{L}_{\text {EA }}$-theory $T$, in order to show $\mathscr{N}_{T} \models T$, it suffices to show $\mathscr{M}_{T^{\oplus}} \models T^{\oplus}$.

Corollary 39 provides a foothold for proving truth of self-referential theories by transfinite induction up to $\omega \cdot \omega$ : in order to prove $\mathscr{N}_{T} \models T$, one can attempt to prove $\mathscr{M}_{T^{\oplus}} \models T^{\oplus} \cap \alpha$ for all $\alpha \in \omega \cdot \omega$ by induction on $\alpha$.

\section{Upward Generic Axioms}

One way to state an epistemological consistency result, for example that a truthful machine can know itself to be true and recursively enumerable, is to show that the schemas in question are consistent with a particular background theory of knowledge. We take a more general approach: show that the doubted schemas are consistent with any background theory satisfying certain conditions.

We say that an $\mathscr{L}_{\mathrm{EA}}$-theory $T$ is $K$-closed if $K \phi \in T$ whenever $\phi \in T$.

Definition 40. Suppose $T_{0}$ is an $\mathscr{L}_{\mathrm{EA}}$-theory. 
1. $T_{0}$ is generic if $\mathscr{N}_{T} \models T_{0}$ for every $\mathscr{L}_{\mathrm{EA}}$-theory $T \supseteq T_{0}$.

2. $T_{0}$ is closed-generic if $T_{0}$ is $K$-closed and $\mathscr{N}_{T} \models T_{0}$ for every $K$-closed $\mathscr{L}_{\mathrm{EA}}$-theory $T \supseteq T_{0}$.

3. $T_{0}$ is r.e.-generic if $T_{0}$ is r.e. and $\mathscr{N}_{T} \models T_{0}$ for every r.e. $\mathscr{L}_{\mathrm{EA}}$-theory $T \supseteq T_{0}$.

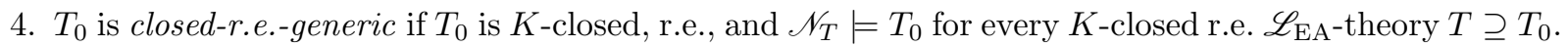

\section{Lemma 41.}

1. Generic+r.e. implies r.e.-generic.

2. Generic $+K$-closed implies closed-generic.

3. Closed-generic+r.e. implies closed-r.e.-generic.

4. R.e.-generic $+K$-closed implies closed-r.e.-generic.

Proof. Straightforward.

Lemma 42. Let $T=\cup_{i \in I} T_{i}$ where each $T_{i}$ is an $\mathscr{L}_{\mathrm{EA} \text {-theory. }}$

1. If the $T_{i}$ are generic, then $T$ is generic.

2. If the $T_{i}$ are closed-generic, then $T$ is closed-generic.

3. If the $T_{i}$ are r.e.-generic and $T$ is r.e., then $T$ is r.e.-generic.

4. If the $T_{i}$ are closed-r.e.-generic and $T$ is r.e., then $T$ is closed-r.e.-generic.

Proof. Straightforward.

Lemma 43. The $\mathscr{L}_{\mathrm{EA}}$-schema $E_{2}$, consisting of $\operatorname{ucl}(K(\phi \rightarrow \psi) \rightarrow K \phi \rightarrow K \psi)$, is generic.

Proof. Suppose $T \supseteq E_{2}$ is arbitrary. For any $\mathscr{L}_{\mathrm{EA}}$-formulas $\phi$ and $\psi$ and assignment $s$, if $\mathscr{N}_{T} \models K(\phi \rightarrow \psi)[s]$ and $\mathscr{N}_{T} \models K \phi[s]$, then

$$
\begin{array}{rlr}
T & \models \phi^{s} \rightarrow \psi^{s} & \text { (Definition 6) } \\
T & \models \phi^{s} & \text { (Definition 6) } \\
T & \models \psi^{s} & \text { (Modus Ponens) } \\
\mathscr{N}_{T} & =K \psi[s], \text { as desired. } & \text { (Definition [6) }
\end{array}
$$

Definition 44. Suppose $T_{0}$ is an $\mathscr{L}_{\mathrm{EA}}$-theory.

1. $T_{0}$ is upgeneric if $\mathscr{M}_{T^{\oplus}} \models T_{0}^{\oplus}$ for every $\mathscr{L}_{\mathrm{EA}}$-theory $T \supseteq T_{0}$.

2. $T_{0}$ is closed-upgeneric if $T_{0}$ is $K$-closed and $\mathscr{M}_{T \oplus} \models T_{0}^{\oplus}$ for every $K$-closed $\mathscr{L}_{\text {EA }}$-theory $T \supseteq T_{0}$.

3. $T_{0}$ is r.e.-upgeneric if $T_{0}$ is r.e. and $\mathscr{M}_{T \oplus} \models T_{0}^{\oplus}$ for every r.e. $\mathscr{L}_{\mathrm{EA}}$-theory $T \supseteq T_{0}$.

4. $T_{0}$ is closed-r.e.-upgeneric if $T_{0}$ is $K$-closed, r.e., and $\mathscr{M}_{T^{\oplus}} \models T_{0}^{\oplus}$ for every $K$-closed r.e. $\mathscr{L}_{\text {EA-theory }}$ $T \supseteq T_{0}$.

Lemma 45. (Compare Lemma 41)

1. Upgeneric $+K$-closed implies closed-generic.

2. Upgeneric+r.e. implies r.e.-upgeneric.

3. Closed-upgeneric+r.e. implies closed-r.e.-upgeneric.

4. R.e.-upgeneric $+K$-closed implies closed-r.e.-upgeneric. 
Proof. Straightforward.

Lemma 46. Suppose $T=\cup_{i \in I} T_{i}$ where the $T_{i}$ are $\mathscr{L}_{\mathrm{EA} \text {-theories. }}$

1. If the $T_{i}$ are upgeneric, then $T$ is upgeneric.

2. If the $T_{i}$ are closed-upgeneric, then $T$ is closed-upgeneric.

3. If the $T_{i}$ are r.e.-upgeneric and $T$ is r.e., then $T$ is r.e.-upgeneric.

4. If the $T_{i}$ are closed-r.e.-upgeneric and $T$ is r.e., then $T$ is closed-r.e.-upgeneric.

Proof. Straightforward.

\section{Lemma 47.}

1. Upgeneric implies generic.

2. Closed-upgeneric implies closed-generic.

3. R.e.-upgeneric implies r.e.-generic.

4. Closed-r.e.-upgeneric implies closed-r.e.-generic.

Proof. By the upward stratification theorem (Theorem 38).

In light of Lemmas 43 and 47, the following shows that upgeneric is strictly stronger than generic.

Lemma 48. $E_{2}$ is not upgeneric. In fact $E_{2}$ is not even closed-r.e.-upgeneric.

Proof. Let $T$ be the smallest $K$-closed $\mathscr{L}_{\mathrm{EA}}$-theory containing the following schemata.

1. $E_{2}$.

2. $K(1=0)$.

3. $K(1=0) \rightarrow(1=0)$.

Since $T \supseteq E_{2}$ is closed r.e., it suffices to exhibit some $\theta \in E_{2}$ and stratifier $\bullet^{+}$such that $\mathscr{M}_{T \oplus} \not \forall \theta^{+}$. If $\bullet+$ is the stratifier given by $X=\{0,1,2, \ldots\}$, the reader can check that

$$
\theta \equiv K(K(1=0) \rightarrow(1=0)) \rightarrow K K(1=0) \rightarrow K(1=0)
$$

works.

Lemma 48 and the following demystify our reason for weakening $E_{2}$ to $E_{2}^{\prime}$.

Lemma 49. The schema $E_{2}^{\prime}$, consisting of ucl $(K(\phi \rightarrow \psi) \rightarrow K \phi \rightarrow K \psi)$ whenever $\operatorname{depth}(\phi) \leq \operatorname{depth}(\psi)$ (Definition 8), is upgeneric.

Proof. Let $T \supseteq E_{2}^{\prime}$ be arbitrary. Suppose $\phi$ and $\psi$ are $\mathscr{L}_{\mathrm{EA}}$-formulas with $\operatorname{depth}(\phi) \leq \operatorname{depth}(\psi)$ and $\bullet+$ is a stratifier, say with

$$
\begin{aligned}
(K \phi)^{+} & \equiv K^{\alpha} \phi^{+} \\
(K \psi)^{+} & \equiv K^{\beta} \psi^{+} \\
(K(\phi \rightarrow \psi))^{+} & \equiv K^{\gamma}\left(\phi^{+} \rightarrow \psi^{+}\right),
\end{aligned}
$$

we will show $\mathscr{M}_{T^{\oplus}}$ satisfies

$$
(\operatorname{ucl}(K(\phi \rightarrow \psi) \rightarrow K \phi \rightarrow K \psi))^{+} \equiv \operatorname{ucl}\left(K^{\gamma}\left(\phi^{+} \rightarrow \psi^{+}\right) \rightarrow K^{\alpha} \phi^{+} \rightarrow K^{\beta} \psi^{+}\right) .
$$


Note that by Lemma 12, $\alpha \leq \beta=\gamma$. Let $s$ be an arbitrary assignment such that $\mathscr{M}_{T \oplus} \models K^{\gamma}\left(\phi^{+} \rightarrow \psi^{+}\right)[s]$ and $\mathscr{M}_{T^{\oplus}}=K^{\alpha} \phi^{+}[s]$. Then

$$
\begin{array}{rlrl}
T^{\oplus} \cap \gamma \models\left(\phi^{+}\right)^{s} \rightarrow\left(\psi^{+}\right)^{s} & \text { (Definition 36) } \\
T^{\oplus} \cap \alpha \models\left(\phi^{+}\right)^{s} & \text { (Definition 36) } \\
T^{\oplus} \cap \beta \models\left(\left(\phi^{+}\right)^{s} \rightarrow\left(\psi^{+}\right)^{s}\right) \wedge\left(\phi^{+}\right)^{s} & \text { (Since } \alpha \leq \beta=\gamma) \\
T^{\oplus} \cap \beta \models\left(\psi^{+}\right)^{s} & \text { (Modus Ponens) } \\
\mathscr{M}_{T^{\oplus}} & =K^{\beta} \psi^{+}[s], \text { as desired. } & \text { (Definition 36) }
\end{array}
$$

Lemma 50. The Assigned Validity schema, consisting of $\phi^{s}$ whenever $\phi$ is valid and $s$ is any assignment, is upgeneric.

Proof. Let $T \supseteq$ (Assigned Validity) be arbitrary. Suppose $\phi$ is valid, $s$ is an assignment, and $\bullet+$ is a stratifier. By Theorem [20, $\phi^{+}$is also valid. Thus $\mathscr{M}_{T^{\oplus}} \models \phi^{+}[s]$, and by Lemma 37, $\mathscr{M}_{T^{\oplus}} \models\left(\phi^{+}\right)^{s}$. By arbitrariness of $\phi, s$, and $\bullet^{+}, \mathscr{M}_{T^{\oplus}} \models(\text { Assigned Validity })^{\oplus}$.

Lemma 51. Any set of true purely arithmetical sentences is upgeneric.

Proof. Trivial: $\mathscr{M}_{T}$ has standard first-order part.

Lemma 52. The schema consisting of the axioms of Epistemic Arithmetic (Peano Arithmetic with induction extended to $\left.\mathscr{L}_{\mathrm{EA}}\right)$ is upgeneric.

Proof. Let $T \supseteq$ (Epistemic Arithmetic). Let $\sigma$ be an axiom of Epistemic Arithmetic, $\bullet^{+}$a stratifier. If $\sigma$ is not an induction instance, then $\mathscr{M}_{T^{\oplus}} \models \sigma^{+}$by Lemma 51] But suppose $\sigma$ is an instance

$$
\operatorname{ucl}(\phi(x \mid 0) \rightarrow \forall x(\phi \rightarrow \phi(x \mid S(x))) \rightarrow \forall x \phi)
$$

of induction, so that $\sigma^{+}$is $\operatorname{ucl}\left(\phi^{+}(x \mid 0) \rightarrow \forall x\left(\phi^{+} \rightarrow \phi^{+}(x \mid S(x))\right) \rightarrow \forall x \phi^{+}\right)$. To show $\mathscr{M}_{T^{\oplus}} \models \sigma^{+}$, let $s$ be an assignment and assume $\mathscr{M}_{T^{\oplus}} \models \phi^{+}(x \mid 0)[s]$ and $\mathscr{M}_{T^{\oplus}} \models \forall x\left(\phi^{+} \rightarrow \phi^{+}(x \mid S(x))\right)[s]$. Then

$$
\begin{aligned}
& \mathscr{M}_{T^{\oplus}} \models \phi^{+}(x \mid 0)^{s} \\
& \mathscr{M}_{T^{\oplus}} \models\left(\phi^{+}\right)^{s(x \mid 0)}
\end{aligned}
$$

(Lemma 37)

$\forall n \in \mathbb{N}$, if $\mathscr{M}_{T^{\oplus}} \models \phi^{+}[s(x \mid n)]$, then $\mathscr{M}_{T^{\oplus}} \models \phi^{+}(x \mid S(x))[s(x \mid n)]$

$\forall n \in \mathbb{N}$, if $\mathscr{M}_{T^{\oplus}} \models\left(\phi^{+}\right)^{s(x \mid n)}$, then $\mathscr{M}_{T^{\oplus}} \models\left(\phi^{+}(x \mid S(x))\right)^{s(x \mid n)}$

$\forall n \in \mathbb{N}$, if $\mathscr{M}_{T^{\oplus}} \models\left(\phi^{+}\right)^{s(x \mid n)}$, then $\mathscr{M}_{T^{\oplus}} \models\left(\phi^{+}\right)^{s(x \mid n+1)}$

$$
\begin{aligned}
\forall n \in \mathbb{N}, \mathscr{M}_{T^{\oplus}} & \models\left(\phi^{+}\right)^{s(x \mid n)} \\
\forall n \in \mathbb{N}, \mathscr{M}_{T^{\oplus}} & \models\left(\phi^{+}\right)[s(x \mid n)] \\
\mathscr{M}_{T^{\oplus}} & \models \forall x \phi^{+}[s], \text { as desired. }
\end{aligned}
$$$$
\text { (Clearly } \psi(x \mid 0)^{s} \equiv \psi^{s(x \mid 0)} \text { ) }
$$

(First-order semantics of $\forall$ and $\rightarrow$ )

(Lemma 37)

$\left(\right.$ Clearly $\left.\psi(x \mid S(x))^{s(x \mid n)} \equiv \psi^{s(x \mid n+1)}\right)$

(Mathematical induction)

(Lemma 37)

(First-order semantics of $\forall$ )

Armed with Lemmas 42 and 46, computations such as Lemmas 43, 49, 50, 51 and 52 can be used as building blocks for background theories of knowledge. Often, schemas we would like as building blocks are not (up)generic in isolation, but become so when paired with other building blocks, as in the following three lemmas.

Lemma 53. $E_{1} \cup$ (Assigned Validity) is upgeneric ( $E_{1}$ consists of $\operatorname{ucl}(K \phi)$ whenever $\phi$ is valid).

Proof. Let $T \supseteq E_{1} \cup$ (Assigned Validity). By Lemma 50, $\mathscr{M}_{T^{\oplus}} \models$ (Assigned Validity) ${ }^{\oplus}$, we need only show $\mathscr{M}_{T^{\oplus}} \models E_{1}^{\oplus}$. Let $\phi$ be valid, $\bullet+$ any stratifier, and $s$ any assignment. Since $T \supseteq$ (Assigned Validity), $T^{\oplus}$ contains the instance

$$
\left(\phi^{s}\right)^{+} \equiv\left(\phi^{+}\right)^{s}
$$

of (Assigned Validity $)^{\oplus}$. In fact, $T^{\oplus} \cap \alpha$ contains $\left(\phi^{+}\right)^{s}$, where $\alpha$ is such that $(K \phi)^{+} \equiv K^{\alpha} \phi^{+}$. Thus by Definition [36, $\mathscr{M}_{T^{\oplus}} \models K^{\alpha} \phi^{+}[s]$, that is, $\mathscr{M}_{T^{\oplus}} \models(K \phi)^{+}[s]$. This shows $\mathscr{M}_{T^{\oplus}} \models E_{1}^{\oplus}$. 
Lemma 54. For any upgeneric $T_{0}, T_{0} \cup K\left(T_{0}\right)$ is upgeneric, where $K\left(T_{0}\right)$ consists of $K \phi$ whenever $\phi \in$ $T_{0}$. Similarly with "upgeneric" replaced by "r.e.-upgeneric", "closed-upgeneric", "closed-r.e.-upgeneric", "generic", "r.e.-generic", "closed-generic", or "closed-r.e.-generic" throughout.

Proof. We prove the upgeneric statement. Suppose $T_{0}$ is upgeneric and $T \supseteq T_{0} \cup K\left(T_{0}\right)$. Since $T_{0}$ is upgeneric and $T \supseteq T_{0}, \mathscr{M}_{T^{\oplus}} \models T_{0}^{\oplus}$. It remains to show $\mathscr{M}_{T \oplus} \models(K \phi)^{+}$for any sentence $\phi \in T_{0}$ and stratifier $\bullet^{+}$. Let $\alpha$ be such that $(K \phi)^{+} \equiv K^{\alpha} \phi^{+}$. By Definition 10, On $\left(\phi^{+}\right) \subseteq \alpha$ and thus $\phi^{+} \in T_{0}^{\oplus} \cap \alpha \subseteq T^{\oplus} \cap \alpha$. Since $T^{\oplus} \cap \alpha \models \phi^{+}, \mathscr{M}_{T^{\oplus}} \models K^{\alpha} \phi^{+}$as desired.

We will not use the following lemma, but it illuminates differences between this paper's upward approach and Carlson's original downward approach.

Lemma 55. $E_{1} \cup E_{2} \cup E_{4} \cup$ (Epistemic Arithmetic) is closed-generic.

Proof. Let $T$ be a $K$-closed theory containing $E_{1}, E_{2}, E_{4}$ and (Epistemic Arithmetic).

By Lemma $43, \mathscr{N}_{T} \models E_{2}$. By Lemmas 52 and $47, \mathscr{N}_{T} \models$ (Epistemic Arithmetic). It remains to show $\mathscr{N}_{T} \models E_{1} \cup E_{4}$. We will show $\mathscr{N}_{T} \models E_{4}$ and sketch $\mathscr{N}_{T} \models E_{1}$.

The typical sentence in $E_{4}$ is $\operatorname{ucl}(K \phi \rightarrow K K \phi)$. Let $s$ be an assignment and assume $\mathscr{N}_{T} \models K \phi[s]$. Then

$$
\begin{aligned}
T & \models \phi^{s} \\
\exists \tau_{1}, \ldots, \tau_{n} \in T & \text { s.t. }\left(\wedge_{i=1}^{n} \tau_{i}\right) \rightarrow \phi^{s} \text { is valid } \\
T & \models K\left(\left(\wedge_{i=1}^{n} \tau_{i}\right) \rightarrow \phi^{s}\right) \\
T & \models\left(\wedge_{i=1}^{n} K\left(\tau_{i}\right)\right) \rightarrow K \phi^{s} \\
T & \models \wedge_{i=1}^{n} K\left(\tau_{i}\right) \\
T & \models K \phi^{s} \\
\mathscr{N}_{T} & \models K K \phi[s] .
\end{aligned}
$$

( $T$ contains $E_{1}$ )

(Repeated applications of $E_{2}$ in $T$ )

( $T$ is $K$-closed)

(Modus Ponens)

(Definition 6)

This shows $\mathscr{N}_{T} \models E_{4}$.

Because of the lack of Assigned Validity, showing $\mathscr{M}_{T} \models E_{1}$ is tricky. We indicate a rough sketch. Carlson's Lemmas 5.23 and 7.1 [6] (pp. 69 \& 72) imply $T \models$ (Assigned Validity) (we invoke Lemma 7.1 with $\mathscr{Q}$ a singleton). As written, Lemma 5.23 demands $T$ also contain $E_{3}$, but it can be shown this is unnecessary. Thus we may assume $T$ contains Assigned Validity. By Lemmas 53 and $47, \mathscr{N}_{T} \models E_{1}$.

Lemma 55 explains why weakening $E_{2}$ to $E_{2}^{\prime}$ required two other seemingly-unrelated weakenings: adding Assigned Validity, and removing $E_{4}$ altogether.

Lemma 56. The Mechanicalness schema,

$$
\operatorname{ucl}\left(\exists e \forall x\left(K \phi \leftrightarrow x \in W_{e}\right)\right) \quad(e \notin \mathrm{FV}(\phi)),
$$

is r.e.-upgeneric.

Proof. Let $T$ be any r.e. $\mathscr{L}_{\mathrm{EA}}$-theory containing the Mechanicalness schema. Let $\bullet{ }^{+}$be a stratifier and let $\alpha$ be such that $(K \phi)^{+} \equiv K^{\alpha} \phi^{+}$. We must show

$$
\mathscr{M}_{T^{\oplus}} \models \operatorname{ucl}\left(\exists e \forall x\left(K^{\alpha} \phi^{+} \leftrightarrow x \in W_{e}\right)\right) .
$$

Let $s$ be any assignment and note

$$
\left\{q \in \mathbb{N}: \mathscr{M}_{T^{\oplus}} \models K^{\alpha} \phi^{+}[s(x \mid q)]\right\}=\left\{q \in \mathbb{N}: T^{\oplus} \cap \alpha \models\left(\phi^{+}\right)^{s(x \mid q)}\right\} .
$$

(Definition [36)

By the Church-Turing Thesis, the latter set is r.e., so there is some $p \in \mathbb{N}$ such that

$$
W_{p}=\left\{q \in \mathbb{N}: \mathscr{M}_{T^{\oplus}} \models K^{\alpha} \phi^{+}[s(x \mid q)]\right\} .
$$


For all $q \in \mathbb{N}$, the following biconditionals are equivalent:

$$
\begin{array}{cr}
\mathscr{M}_{T^{\oplus}} \models K^{\alpha} \phi^{+} \leftrightarrow x \in W_{e}[s(e \mid p)(x \mid q)] & \\
\mathscr{M}_{T^{\oplus}} \models K^{\alpha} \phi^{+}[s(e \mid p)(x \mid q)] \text { iff } \mathscr{M}_{T^{\oplus} \models x \in W_{e}[s(e \mid p)(x \mid q)]} & \text { (First-order semantics of } \leftrightarrow \text { ) } \\
\mathscr{M}_{T^{\oplus}} \models K^{\alpha} \phi^{+}[s(x \mid q)] \text { iff } \mathscr{M}_{T^{\oplus}} \models x \in W_{e}[s(e \mid p)(x \mid q)] & \text { (Since } e \notin \mathrm{FV}(\phi)) \\
\mathscr{M}_{T^{\oplus}} \models K^{\alpha} \phi^{+}[s(x \mid q)] \text { iff } q \in W_{p} . & \text { (Since } \mathscr{M}_{T^{\oplus}} \text { has standard first-order part) }
\end{array}
$$

The latter is true by definition of $p$. By arbitrariness of $q, \mathscr{M}_{T^{\oplus}} \models \exists e \forall x\left(K^{\alpha} \phi^{+} \leftrightarrow x \in W_{e}\right)[s]$.

Corollary 57. (Recall the definition of $T_{\mathrm{SMT}}^{w}$ from the end of Section 2) Let $\left(T_{\mathrm{SMT}}^{w}\right) \backslash E_{3}$ be the smallest $K$-closed theory containing $E_{1}$, Assigned Validity, $E_{2}^{\prime}$, Epistemic Arithmetic, and Mechanicalness. (Loosely speaking, $T_{\mathrm{SMT}}^{w}$ minus $E_{3}$.) Then $\left(T_{\mathrm{SMT}}^{w}\right) \backslash E_{3}$ is r.e.-upgeneric.

\section{The Main Result}

With the machinery of Section 5, we are able to state our main result in a generalized form. Informally:

An r.e.-upgeneric theory remains true upon augmentation by knowledge of its own truthfulness.

Reinhardt's conjecture (proved by Carlson) was that the Strong Mechanistic Thesis is consistent with a particular background theory of knowledge. We showed (Lemma 56) that Mechanicalness is r.e.-upgeneric. By Lemma 54, the pair consisting of Mechanicalness and the Strong Mechanistic Thesis, is r.e.-upgeneric. Thus as long as the background theory of knowledge is r.e. and built of r.e.-generic pieces along with truthfulness, the corresponding conjecture is a special case of this main result.

Recall (Definition 6) that an $\mathscr{L}_{\mathrm{EA}}$-theory $T$ is true if $\mathscr{N}_{T} \models T$.

Theorem 58. Let $T_{0}$ be an r.e.-upgeneric $\mathscr{L}_{\mathrm{EA}}$-theory. Let $T_{1}$ be $T_{0} \cup E_{3}$, that is, $T_{0}$ along with all axioms of the form ucl $(K \phi \rightarrow \phi)$. Let $T$ be the smallest $K$-closed theory containing $T_{1}$. Then $T$ is true.

Proof. By Corollary 39 it is enough to show $\mathscr{M}_{T^{\oplus}} \models T^{\oplus}$. We will use transfinite induction up to $\omega \cdot \omega$ to show that for all $\alpha \in \omega \cdot \omega, \mathscr{M}_{T \oplus} \models T^{\oplus} \cap \alpha$.

Let $\sigma \in T^{\oplus} \cap \alpha$. Then $\sigma \equiv \theta^{+}$for some $\theta \in T$ and some stratifier $\bullet^{+}$. We will show $\mathscr{M}_{T^{\oplus}} \models \theta^{+}$.

Case 1: $\theta \in T_{0}$. Then $\mathscr{M}_{T^{\oplus}} \models \theta^{+}$because $T \supseteq T_{0}$ is r.e. and $T_{0}$ is r.e.-upgeneric.

Case 2: $\theta$ is $K \phi$ for some sentence $\phi \in T$. Let $\alpha_{0}$ be such that $(K \phi)^{+} \equiv K^{\alpha_{0}} \phi^{+}$. By Definition 10 , $\operatorname{On}\left(\phi^{+}\right) \subseteq \alpha_{0}$ and thus $\phi^{+} \in T^{\oplus} \cap \alpha_{0}$, so $T^{\oplus} \cap \alpha_{0} \models \phi^{+}$, so $\mathscr{M}_{T^{\oplus}} \models K^{\alpha_{0}} \phi^{+}$.

Case 3: $\theta$ is $\operatorname{ucl}(K \phi \rightarrow \phi)$ for some $\phi$. Let $\alpha_{0}$ be such that $(K \phi)^{+} \equiv K^{\alpha_{0}} \phi^{+}$, so $\theta^{+}$is $\operatorname{ucl}\left(K^{\alpha_{0}} \phi^{+} \rightarrow \phi^{+}\right)$. Since $\theta^{+} \in T^{\oplus} \cap \alpha$, this forces $\alpha_{0}<\alpha$. Let $s$ be any assignment and assume $\mathscr{M}_{T^{\oplus}} \models K^{\alpha_{0}} \phi^{+}[s]$. Then:

$$
\begin{array}{rlrl}
\mathscr{M}_{T^{\oplus}} & =K^{\alpha_{0}} \phi^{+}[s] & \text { (Assumption) } \\
T^{\oplus} \cap \alpha_{0} & \models\left(\phi^{+}\right)^{s} & \text { (Definition 吕) } \\
\mathscr{M}_{T^{\oplus}} & \models\left(\phi^{+}\right)^{s} & & \text { (Lemma 37) }
\end{array}
$$

Corollary 59. $T_{\mathrm{SMT}}^{w}$ is true.

Proof. By Theorem 58 and Corollary 57.

If one is willing to induct up to $\epsilon_{0} \cdot \omega$ and use machinery from [5], it is possible (without the grievous sacrifices we have made in this paper) to generalize Reinhardt's conjecture to a statement of the form:

Any r.e. theory that is generic in a very specific sense (one that allows $E_{2}$ as building block) remains true upon augmentation by knowledge of its own truthfulness. $(*)$ 
The specific notion of "generic" in order for this to work is somewhat complicated and hinges on [5], putting it out of the present paper's scope. It does admit Mechanicalness as building block, so that $(*)$ really is a generalization of Reinhardt's conjecture, and the notion also admits full $E_{2}$, which in turn allows building blocks containing $E_{4}$.

The main result of [2] can also be generalized in this manner. The methods of that paper are easily modified to prove:

For any r.e. $\mathscr{L}_{\mathrm{EA}}$-theory $T$ that is generic (in the sense of Definition 40), there is an $n \in \mathbb{N}$ such that $T^{\prime}$ is true, where $T^{\prime}$ is the smallest $K$-closed theory containing $T$ along with the schema $\forall x\left(K \phi \leftrightarrow\langle x, \overline{\ulcorner\phi\urcorner}\rangle \in W_{\bar{n}}\right)(\mathrm{FV}(\phi) \subseteq\{x\})$. Less formally, any such generic knowing machine can be taught its own code and still remain true.

One possible application of this paper is to reverse mathematics [14]. Since the results (except Lemma [52) only use induction up to $\omega \cdot \omega$, suitable versions (minus Lemma 52 and references to $\mathbb{N}$ ) could be formalized and proved in weak subsystems of arithmetic.

\section{References}

[1] Alexander, S. (2013). The Theory of Several Knowing Machines. Doctoral dissertation, the Ohio State University.

[2] Alexander, S. (preprint). A machine that knows its own code. To appear in Studia Logica.

[3] Alexander, S. (preprint). Self-referential theories. Submitted.

[4] Benacerraf, P. (1967). God, the Devil, and Gödel. The Monist, 51, 9-32.

[5] Carlson, T.J. (1999). Ordinal arithmetic and $\Sigma_{1}$-elementarity. Archive for Mathematical Logic, 38, 449460.

[6] Carlson, T.J. (2000). Knowledge, machines, and the consistency of Reinhardt's strong mechanistic thesis. Annals of Pure and Applied Logic, 105, 51-82.

[7] Carlson, T.J. (2001). Elementary patterns of resemblance. Annals of Pure and Applied Logic, 108, $19-77$.

[8] Carlson, T.J. (2012). Sound Epistemic Theories and Collapsing Knowledge. Slides from the Workshop on The Limits and Scope of Mathematical Knowledge at the University of Bristol.

[9] Lucas, J.R. (1961). Minds, machines, and Gödel. Philosophy, 36, 112-127.

[10] Penrose, R. (1989). The Emperor's New Mind: Concerning Computers, Minds, and the Laws of Physics. Oxford University Press.

[11] Putnam, H. (2006). After Gödel. Logic Journal of the IGPL, 14, 745-754.

[12] Reinhardt, W. (1985). Absolute versions of incompleteness theorems. Noûs, 19, 317-346.

[13] Shapiro, S. (1985). Epistemic and Intuitionistic Arithmetic. In: S. Shapiro (ed.), Intensional Mathematics (North-Holland, Amsterdam), pp. 11-46.

[14] Simpson, S. (2009). Subsystems of Second Order Arithmetic. 2nd Edition, Cambridge University Press. 